Case Report

\title{
Postmenopausal Deep Infiltrating Endometriosis of the Colon: Rare Location and Novel Medical Therapy
}

\author{
Benjamin M. Snyder (D), ${ }^{1}$ Joseph W. Beets, ${ }^{2}$ Bruce A. Lessey, ${ }^{3}$ \\ Samuel R. W. Horton, ${ }^{4}$ and Gary A. Abrams ${ }^{2}$ \\ ${ }^{1}$ University of South Carolina School of Medicine Greenville, Greenville, SC, USA \\ ${ }^{2}$ Department of Gastroenterology/Liver Center, Greenville Health System, University of South Carolina School of Medicine Greenville, \\ Greenville, SC, USA \\ ${ }^{3}$ Department of Obstetrics and Gynecology, Greenville Health System, University of South Carolina School of Medicine Greenville, \\ Greenville, SC, USA \\ ${ }^{4}$ Department of Pathology, Greenville Health System, University of South Carolina School of Medicine Greenville, Greenville, SC, USA
}

Correspondence should be addressed to Benjamin M. Snyder; bmsnyder@email.sc.edu

Received 13 November 2017; Accepted 18 January 2018; Published 14 February 2018

Academic Editor: Daniel C. Damin

Copyright (C) 2018 Benjamin M. Snyder et al. This is an open access article distributed under the Creative Commons Attribution License, which permits unrestricted use, distribution, and reproduction in any medium, provided the original work is properly cited.

We report an uncommon case of deep infiltrating endometriosis of the colon presenting as iron deficiency anemia nine years after hysterectomy with bilateral salpingo-oophorectomy. The endometrial implant was found at the hepatic flexure, an exceedingly rare location for endometriosis invasion with no cases distinctly reported in the literature. Additionally, the presentation of gastrointestinal endometriosis as iron deficiency anemia is not well documented in the literature. Instead of surgery, we prescribed a novel medical therapeutic approach using conjugated estrogen-bazedoxifene to antagonize the proliferative effects of estrogen on endometrial tissue. After five months of therapy and repeat colonoscopy, no evidence of endometrial tissue remained in the hepatic flexure.

\section{Introduction}

Endometriosis is a common disorder in women of reproductive age occurring in 8-10\% [1]. However, endometriosis has not been significantly studied in postmenopausal women. The development of endometriosis after menopause is a rare phenomenon, and it has often been reported in the setting of hormone replacement therapy (HRT) [2] and treatment with tamoxifen [3, 4]. While postmenopausal endometriosis is uncommon, deep infiltrating endometriosis involving the colon in postmenopausal women has been noted only in the case report literature [5-9]. We describe a case of a postmenopausal woman presenting with iron deficiency anemia due to colonic endometrial infiltration and a novel therapeutic approach.

\section{Case Report}

A 60-year-old Caucasian woman was referred to gastroenterology department due to three years of persistent iron deficiency anemia (IDA) resistant to oral iron supplementation with patient-reported blood in the stool. Her history was significant for endometriosis in early reproductive years and uterine fibroids treated by hysterectomy with bilateral salpingo-oophorectomy nine years before her current presentation. She was given oral estradiol therapy as part of hormone replacement therapy (HRT). She underwent an esophagogastroduodenoscopy (EGD) that was of normal result and a colonoscopy that revealed moderately severe diverticulosis and a nodular ulcerated lesion at the hepatic flexure (Figure 1) that was partially removed by hot snare cauterization (Figure 2). This was not a complete resection and allowed for better tissue sampling. The nodule appeared 


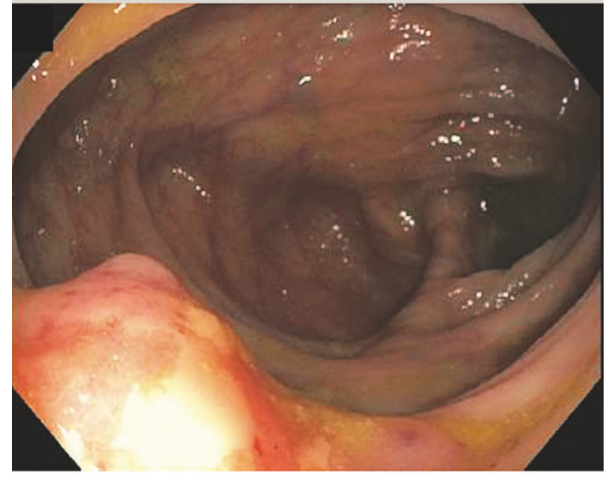

FIGURE 1: The nodule was visualized at the hepatic flexure with mild ulceration and bleeding at the surface. Ulceration and blood loss were the presumed explanation for the patient's chronic iron deficiency.

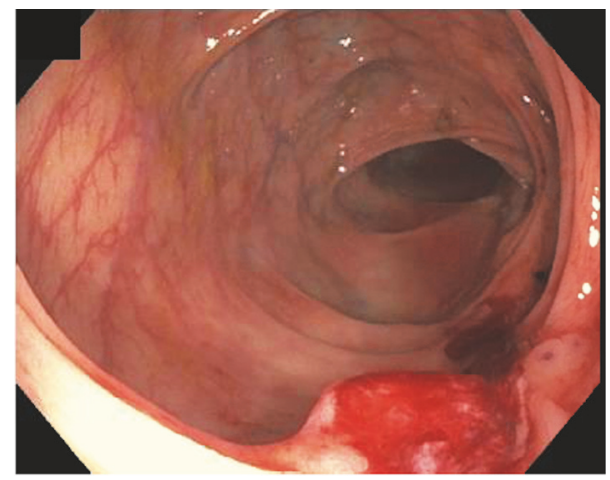

FIGURE 2: The nodule was partially removed by cold snare polypectomy but was not amenable to complete removal under endoscopy.

deeper than what could be visualized by endoscopy and was biopsied multiple times. India ink was used to tattoo the location of the nodule for a follow-up examination.

Histological analysis of the biopsies noted mural endometriosis and adenomatous tissue within the endometrial tissue and surrounding mucosa (Figure 3). The endometrial tissue infiltrated the full thickness of the biopsy specimen, which extended through the submucosa. The presence of endometrial tissue through the depth of the biopsy, extending into the mucosa, is indicative of complete thickness penetration of the colonic wall. Though the patient experienced no other symptoms of endometriosis, deep infiltrating endometriosis was a likely explanation for her IDA. Gynecology was consulted, and the patient's estradiol therapy was discontinued. Given that the subject had few physical symptoms of her disease but had previously suffered vasomotor symptoms of menopause, medical treatment was an appropriate approach for her condition. A course of conjugated estrogenbazedoxifene (CE/BZA) (Duavee: 0.45-20 mg, Pfizer, New York, NY) was selected instead of surgery. CE/BZA is commonly used in women with a uterus who desire HRT, as it prevents estrogen from stimulating endometrial tissues using the selective estrogen receptor modulator, bazedoxifene.
Approximately three months after her original visit to gastroenterology department and consultation with hematology, the patient received two intravenous iron infusions one week apart in conjunction with the course of CE/BZA therapy. Follow-up colonoscopy, eight months after the initial colonoscopy and five months of CE/BZA therapy, demonstrated scarring from the initial procedure and no residual nodule at the hepatic flexure (Figure 4). Biopsies taken identified fragments of tubular adenoma without any evidence of remaining endometriosis suggesting complete regression. Follow-up labs revealed normalization of iron studies, anemia, and microcytosis. Iron infusion and complete regression of endometrial tissue and reversal of minor blood loss from associated ulceration are the presumptive explanation for resolution of this patient's iron deficiency anemia.

\section{Discussion}

Deep endometriosis (also called deep infiltrating endometriosis) is a form of endometriosis that invades any vital structures such as bowel, ureters, or bladder [10]. Intestinal involvement of endometriosis has been estimated to exist in between $3.8 \%$ and $37 \%$ of patients with an endometriosis diagnosis [11]. The most common sites of bowel endometriosis are rectum, ileum, appendix, and cecum from most to least prevalent, while some cases of gastric and transverse colonic involvement have been reported [11]. Endometriosis is often difficult to diagnose due to the generalized nature of symptoms: pelvic pain, dysmenorrhea, dyspareunia, and dysuria/dyschezia [12]. Symptoms associated with bowel endometriosis are abdominal pain relieved with defection, change in frequency or appearance of stool, and abnormal rectal bleeding, but most of these symptoms are seen only in cases of mucosal involvement [13]. Additionally, it is difficult to diagnose bowel endometriosis even with colonoscopy as most cases do not infiltrate beyond the serosa and very few infiltrate the mucosa [14]. With a wide range of presentations and the often asymptomatic disease course, until it significantly progressed, deep infiltrating endometriosis is a complicated disease to diagnose and treat.

The treatment of endometriosis has primarily focused on alleviating symptoms and restoring fertility in women of reproductive age [15-17], but to this point, no curative therapy exists. To date, research has primarily focused on the surgical treatment of deep endometriosis with fewer resources being devoted to understanding the medical management of the disease. Specifically, research in postmenopausal endometriosis has purported to indicate the necessity of resection due to the risk of malignancy [18, 19]. However, recurrence of the disease is possible even with surgical intervention, and there is a risk of significant complications as a result of surgical resection especially in bowel endometriosis [20]. There has been a rise in invasive bowel resection for treatment of colorectal endometriosis in recent years, and some authors have begun to question the efficacy of such a practice in favor of less invasive interventions such as ablation or medical management [2123]. Complete excision of endometriosis of the bowel, more 


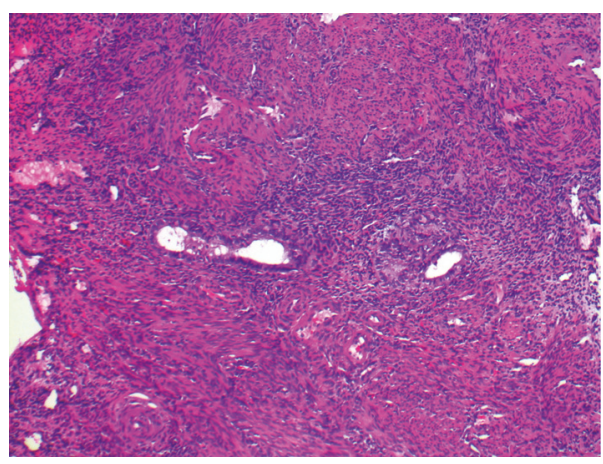

(a)

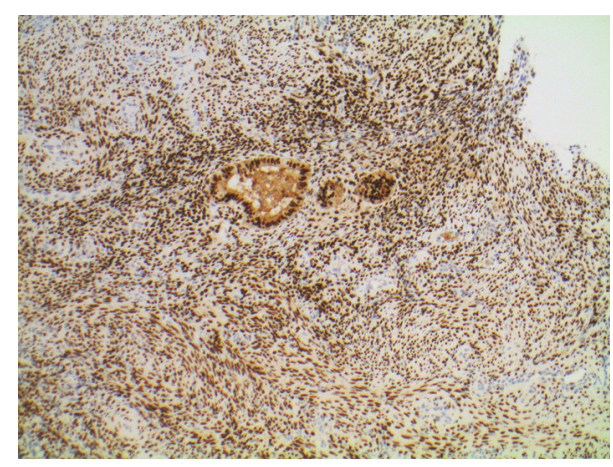

(b)

FIGURE 3: (a) Staining of the colonic nodule shows active proliferative endometrial glands and stromal tissue (hematoxylin and eosin, 40x). (b) Immunohistochemistry demonstrates diffusely positive staining for the estrogen receptor in a pattern consistent with endometrial tissue $(100 x)$.

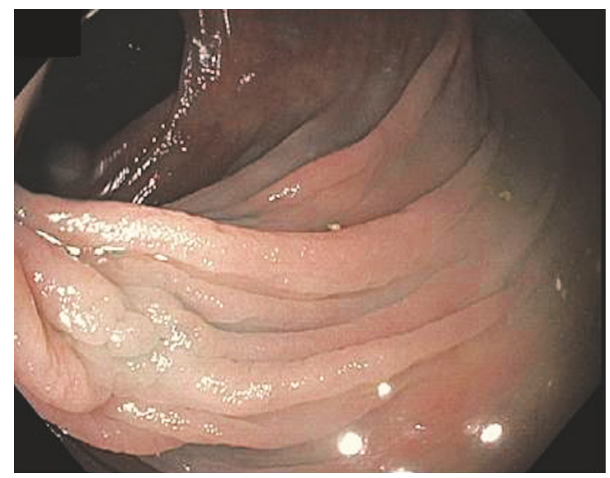

FIGURE 4: Approximately eight months after cold snare polypectomy and treatment with conjugated estrogen/bazedoxifene, no evidence of the nodule remained at the marked site at the hepatic flexure of the colon.

than ablation, has been shown to reduce some symptoms such as dyspareunia, but overall symptoms are decreased nearly identically with ablation or excision [24]. The difficulty of completely eliminating endometriosis of the bowel and permanently reducing symptoms is the preferential association of endometriosis tissue with the enteric nervous system, which also accounts for the set of symptoms seen in deep infiltrating endometriosis of the bowel $[13,25$, 26]. Additionally, macronodules of endometriosis may be readily visible during surgery, but micronodules are identified in histology making complete excision of endometriosis unlikely [27]. As discussed here, many complicating factors are associated with surgical intervention in deep infiltrating endometriosis in a postmenopausal population. A more patient-oriented approach evaluates specific patient factors as in this case, demonstrating a less invasive method, that is, ablation followed by CE/BZA therapy in this case.

Medical management of endometriosis and especially postmenopausal endometriosis is evolving. The currently postulated mechanisms of premenopausal endometriosis include retrograde menstruation, coelomic metaplasia, immune deficiencies, and Mullerian remnants, but these do not adequately account for all cases in women of reproductive age [17]. Postmenopausal endometriosis cannot be fully explained by the postulated mechanisms, but high levels of ectopic estrogen production from nonovarian tissue can activate endometrial tissue [28]. Aromatase has been shown to have a role in the pathogenesis of endometriosis and has long been a proposed target for treatment [29]. Few cases of the treatment of postmenopausal endometriosis with aromatase inhibitors (AI) have been reported; however they suggest that significant symptom reduction is possible with AIs [18]. One case even notes the positive effects of AIs on abdominal endometriosis and suggests a potential role for AIs in the treatment of postmenopausal deep infiltrating endometriosis [30]. Although there are articles demonstrating the usefulness of AIs in postmenopausal endometriosis, empiric research is lacking.

CE/BZA was approved in 2013 for treatment of the vasomotor symptoms associated with menopause as well as the treatment of postmenopausal osteoporosis by a nonhormone replacement therapy/menopausal hormone therapy mechanism [31]. CE/BZA is a combination of conjugated estrogen and bazedoxifene, a selective estrogen receptor modulator (SERM) that induces degradation of estrogen receptors in breast and endometrial tissue [32]. Original clinical trials validated the efficacy of $\mathrm{CE} / \mathrm{BZA}$ in reduction of menopausal vasomotor symptoms and protection against osteoporosis, while demonstrating the protective effects of bazedoxifene on breast and endometrial tissue in the presence of estrogen [33]. Research has also shown that $\mathrm{CE} / \mathrm{BZA}$ can cause regression of endometriosis in a murine model $[34,35]$. It was by this mechanism hypothesized that CE/BZA would be an ideal option for medical management in this postmenopausal patient thereby inducing regression of endometrial tissue while providing the protective bone effects of estrogen and preventing vasomotor symptoms of menopause.

In conclusion, this case highlights an unusual presentation of iron deficiency anemia due to a rare colonic location of deep infiltrating endometriosis in a postmenopausal woman 
successfully treated medically with a novel and less invasive approach (CE/BZA) that provides mechanisms for both symptom control and disease regression.

\section{Consent}

Informed consent was obtained from the patient discussed in this case report.

\section{Disclosure}

Gary A. Abrams is the article guarantor. The source is Greenville Memorial Hospital, Greenville, SC.

\section{Conflicts of Interest}

The authors declare that there are no conflicts of interest regarding the publication of this paper.

\section{Acknowledgments}

Funding for this submission is provided by the Department of Medicine at Greenville Memorial Hospital.

\section{References}

[1] P. Acién and I. Velasco, "Endometriosis: A Disease That Remains Enigmatic," ISRN Obstetrics and Gynecology, vol. 2013, pp. 1-12, 2013.

[2] Sung Woo Choi, Hye Nam Lee, Seok Jin Kang, and Hyung Ok Kim, "A case of cutaneous endometriosis developed in postmenopausal woman receiving hormonal replacement," Journal of the American Academy of Dermatology, vol. 41, no. 2, pp. 327329, 1999.

[3] C. H. Buckley, "Tamoxifen-associated postmenopausal endometriosis," Histopathology, vol. 31, no. 3, p. 296, 1997.

[4] I. Cohen, M. M. Altaras, S. Lew, R. Tepper, Y. Beyth, and G. BenBaruch, "Ovarian Endometrioid Carcinoma and Endometriosis Developing in a Postmenopausal Breast Cancer Patient during Tamoxifen Therapy: A Case Report and Review of the Literature," Gynecologic Oncology, vol. 55, no. 3, pp. 443-447, 1994.

[5] B. Deval, A. Rafii, M. F. Dachez, R. Kermanash, and M. Levardon, "Sigmoid endometriosis in a postmenopausal woman," American Journal of Obstetrics \& Gynecology, vol. 187, no. 6, pp. 1723-1725, 2002.

[6] K. Izuishi, T. Sano, A. Shiota, H. Mori, and K. Ebara, "Small bowel obstruction caused by endometriosis in a postmenopausal woman," Asian Journal of Endoscopic Surgery, vol. 8, no. 2, pp. 205-208, 2015.

[7] V. E. Klenov, T. A. Potretzke, J. K. Sehn, and P. H. Thaker, "Postmenopausal invasive endometriosis requiring supralevator pelvic exenteration," Obstetrics \& Gynecology, vol. 126, no. 6, pp. 1215-1218, 2015.

[8] J. G. Petros, N. Spirito, and R. Gosshein, "Endometriosis causing colon obstruction in two postmenopausal women," Mount Sinai Journal of Medicine, vol. 59, no. 4, pp. 362-365, 1992.

[9] P. Popoutchi, C. R. dos Reis Lemos, J. C. R. Silva, A. A. Nogueira, O. Feres, and J. J. R. da Rocha, "Postmenopausal intestinal obstructive endometriosis: Case report and review of the literature," São Paulo Medical Journal, vol. 126, no. 3, pp. 190193, 2008.
[10] P. Vercellini, G. Frontino, G. Pietropaolo, U. Gattei, R. Daguati, and P. G. Crosignani, "Deep endometriosis: Definition, pathogenesis, and clinical management," The Journal of Minimally Invasive Gynecology , vol. 11, no. 2, pp. 153-161, 2004.

[11] V. Remorgida, S. Ferrero, E. Fulcheri, N. Ragni, and D. C. Martin, "Bowel endometriosis: presentation, diagnosis, and treatment," Obstetrical \& Gynecological Survey, vol. 62, no. 7, pp. 461-470, 2007.

[12] J. Nassif, P. Trompoukis, S. Barata, A. Furtado, B. Gabriel, and A. Wattiez, "Management of deep endometriosis," Reproductive BioMedicine Online, vol. 23, no. 1, pp. 25-33, 2011.

[13] V. Remorgida, N. Ragni, S. Ferrero, P. Anserini, P. Torelli, and E. Fulcheri, "The involvement of the interstitial Cajal cells and the enteric nervous system in bowel endometriosis," Human Reproduction, vol. 20, no. 1, pp. 264-271, 2005.

[14] M. Milone, A. Mollo, M. Musella et al., "Role of colonoscopy in the diagnostic work-up of bowel endometriosis," World Journal of Gastroenterology, vol. 21, no. 16, pp. 4997-5001, 2015.

[15] C. Mohr, F. R. Nezhat, C. H. Nezhat, D. S. Seidman, and C. R. Nezhat, "Fertility considerations in laparoscopic treatment of infiltrative bowel endometriosis.," JSLS : Journal of the Society of Laparoendoscopic Surgeons / Society of Laparoendoscopic Surgeons, vol. 9, no. 1, pp. 16-24, 2005.

[16] A. Moini, L. Bahar, M. Ashrafinia, B. Eslami, R. Hosseini, and N. Ashrafinia, "Fertility outcome after operative laparoscopy versus no treatment in infertile women with minimal or mild endometriosis," International Journal of Fertility \& Sterility, vol. 5, no. 4, pp. 235-240, 2012.

[17] P. Vercellini, P. Viganò, E. Somigliana, and L. Fedele, "Endometriosis: pathogenesis and treatment," Nature Reviews Endocrinology, vol. 10, no. 5, pp. 261-275, 2014.

[18] N. P. Polyzos, H. M. Fatemi, A. Zavos et al., "Aromatase inhibitors in post-menopausal endometriosis," Reproductive Biology and Endocrinology, vol. 9, article no. 90, 2011.

[19] L. H. Thomsen, T. H. Schnack, K. Buchardi et al., "Risk factors of epithelial ovarian carcinomas among women with endometriosis: a systematic review," Acta Obstetricia et Gynecologica Scandinavica, vol. 96, no. 6, pp. 761-778, 2017.

[20] C. Meuleman, C. Tomassetti, A. D'Hoore et al., "Surgical treatment of deeply infiltrating endometriosis with colorectal involvement," Human Reproduction Update, vol. 17, no. 3, pp. 311-326, 2011.

[21] M. S. Abrão, F. Petraglia, T. Falcone, J. Keckstein, Y. Osuga, and C. Chapron, "Deep endometriosis infiltrating the rectosigmoid: Critical factors to consider before management," Human Reproduction Update, vol. 21, no. 3, pp. 329-339, 2015.

[22] P. Acién, C. Núñez, F. Quereda, I. Velasco, M. Valiente, and V. Vidal, "Is a bowel resection necessary for deep endometriosis with rectovaginal or colorectal involvement?" International Journal of Women's Health, vol. 5, no. 1, pp. 449-455, 2013.

[23] P. Vercellini, P. G. Crosignani, E. Somigliana, N. Berlanda, G. Barbara, and L. Fedele, "Medical treatment for rectovaginal endometriosis: what is the evidence?" Human Reproduction, vol. 24, no. 10, pp. 2504-2514, 2009.

[24] M. Healey, C. Cheng, and H. Kaur, "To excise or ablate endometriosis? A prospective randomized double-blinded trial after 5-year follow-up," Journal of Minimally Invasive Gynecology, vol. 21, no. 6, pp. 999-1004, 2014.

[25] V. Anaf, I. E. Nakadi, P. Simon et al., "Preferential infiltration of large bowel endometriosis along the nerves of the colon," Human Reproduction, vol. 19, no. 4, pp. 996-1002, 2004. 
[26] G. Wang, N. Tokushige, P. Russell, S. Dubinovsky, R. Markham, and I. S. Fraser, "Hyperinnervation in Intestinal Deep Infiltrating Endometriosis," Journal of Minimally Invasive Gynecology, vol. 16, no. 6, pp. 713-719, 2009.

[27] A. Badescu, H. Roman, M. Aziz et al., "Mapping of bowel occult microscopic endometriosis implants surrounding deep endometriosis nodules infiltrating the bowel," Fertility and Sterility, vol. 105, no. 2, pp. 430-434, 2016.

[28] C. L. Bendon and C. M. Becker, "Potential mechanisms of postmenopausal endometriosis," Maturitas, vol. 72, no. 3, pp. 214-219, 2012.

[29] K. M. Zeitoun and S. E. Bulun, "Aromatase: a key molecule in the pathophysiology of endometriosis and a therapeutic target," Fertility and Sterility, vol. 72, no. 6, pp. 961-969, 1999.

[30] I. E. Sasson and H. S. Taylor, "Aromatase inhibitor for treatment of a recurrent abdominal wall endometrioma in a postmenopausal woman," Fertility and Sterility, vol. 92, no. 3, pp. 1170-e4, 2009.

[31] T. Goldberg and B. Fidler, "Conjugated Estrogens/Bazedoxifene(Duavee) A Novel Agent for the Treatment of Moderate-toSevere Vasomotor Symptoms Associated with Menopause and the Prevention of Postmenopausal Osteoporosis," P\&T, vol. 40, no. 3, pp. 178-182, 2015.

[32] R. Kagan, S. R. Goldstein, J. H. Pickar, and B. S. Komm, "Patient considerations in the management of menopausal symptoms: Role of conjugated estrogens with bazedoxifene," Therapeutics and Clinical Risk Management, vol. 12, pp. 549-562, 2016.

[33] D. F. Archer, E. W. Freeman, B. S. Komm et al., "Pooled Analysis of the Effects of Conjugated Estrogens/Bazedoxifene on Vasomotor Symptoms in the Selective Estrogens, Menopause, and Response to Therapy Trials," Journal of Women's Health, vol. 25, no. 11, pp. 1102-1111, 2016.

[34] H. Naqvi, S. Sakr, T. Presti, G. Krikun, B. Komm, and H. S. Taylor, "Treatment with bazedoxifene and conjugated estrogens results in regression of endometriosis in a murine model," Biology of Reproduction, vol. 90, no. 6, article no. 121, 2014.

[35] S. Sakr, H. Naqvi, B. Komm, and H. S. Taylor, "Endometriosis impairs bone marrow-derived stem cell recruitment to the uterus whereas bazedoxifene treatment leads to endometriosis regression and improved uterine stem cell engraftment," Endocrinology, vol. 155, no. 4, pp. 1489-1497, 2014. 


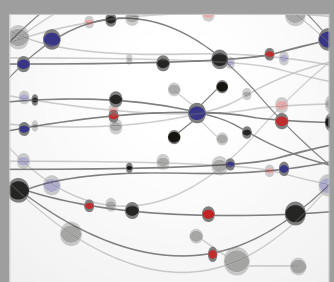

The Scientific World Journal
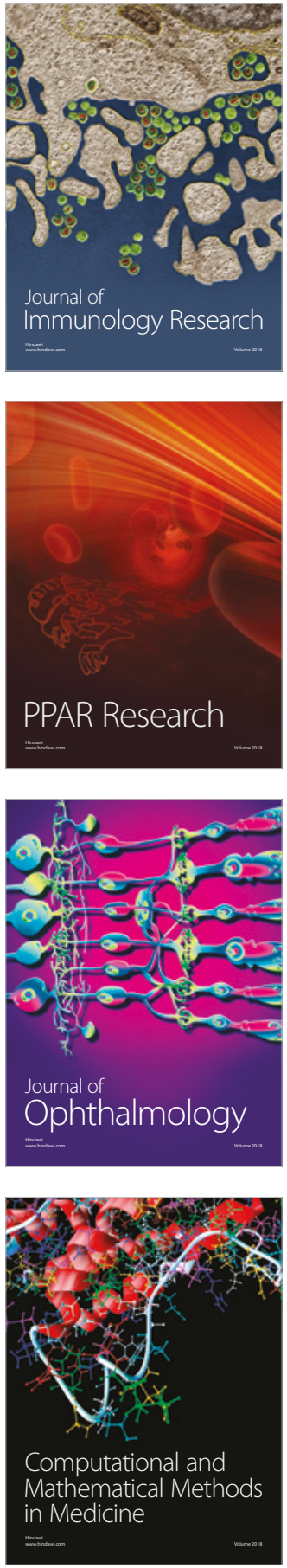

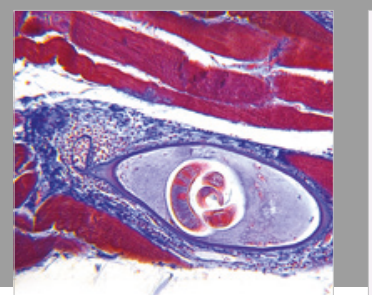

Gastroenterology Research and Practice

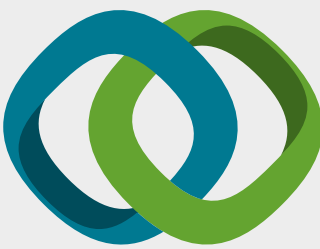

\section{Hindawi}

Submit your manuscripts at

www.hindawi.com
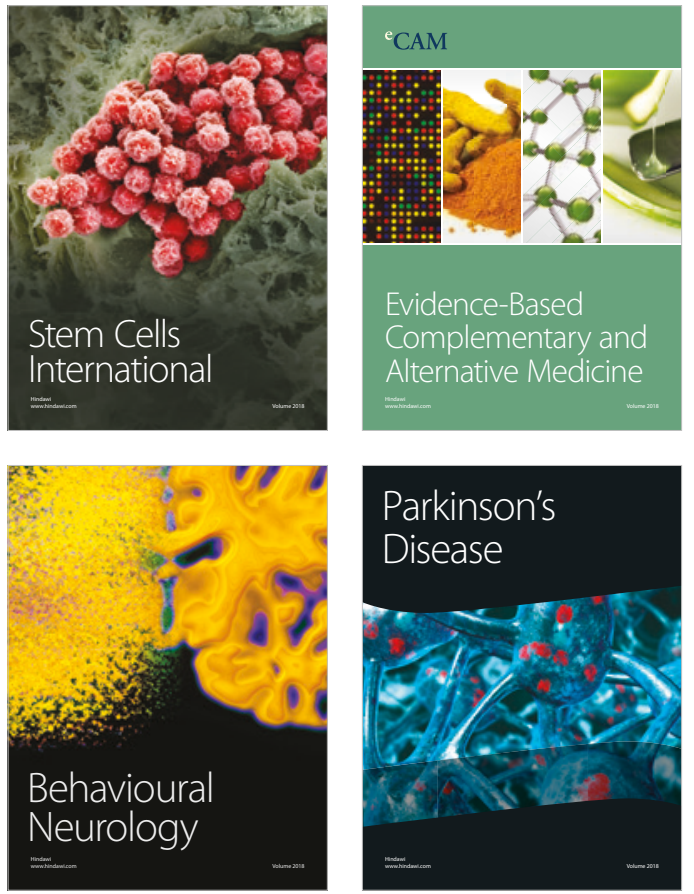

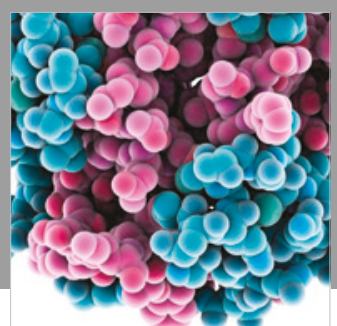

ournal of

Diabetes Research

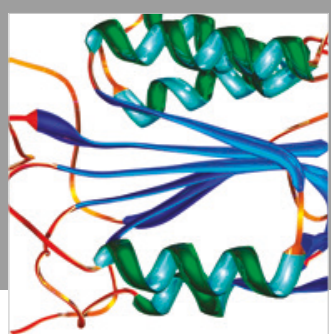

Disease Markers
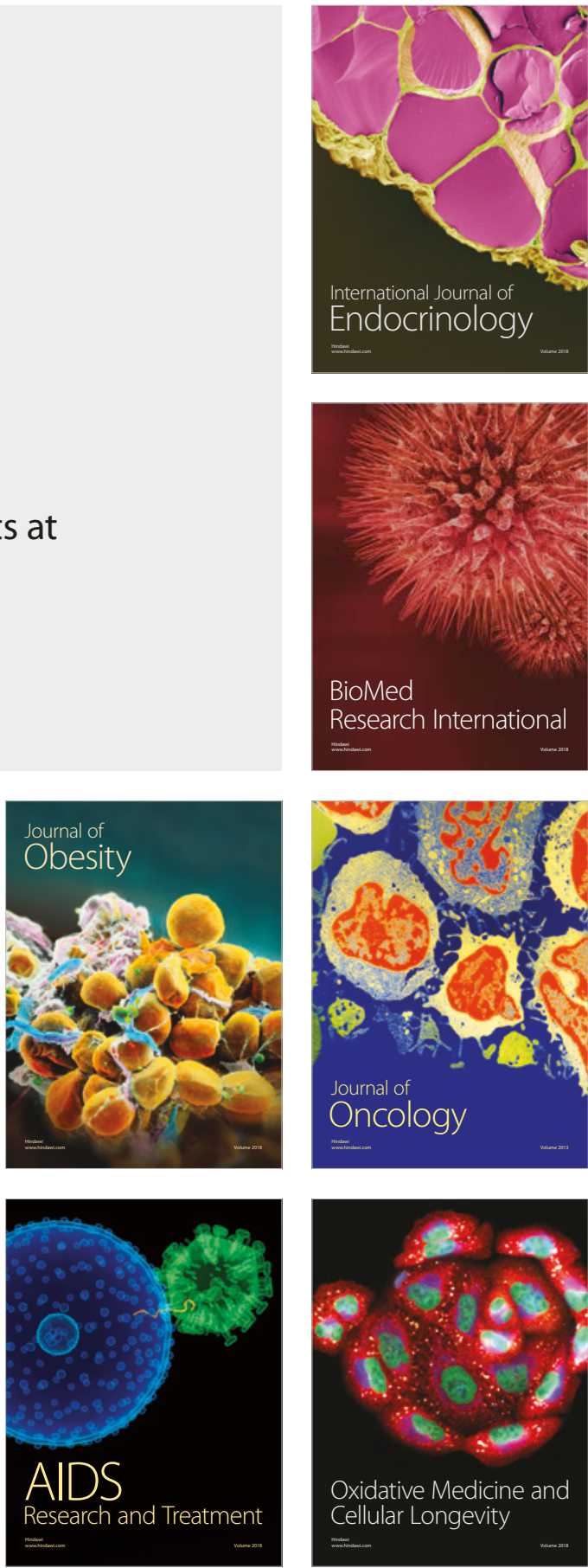